\title{
Probabilistic Approach to Damage Tolerance Design of Aircraft Composite Structures
}

\author{
Kuen Y. Lin ${ }^{*}$ and Andrey V. Styuart ${ }^{\dagger}$ \\ University of Washington, Seattle, WA, 98195-2400
}

\begin{abstract}
A probabilistic approach to determine design parameters for damage-tolerant composite aircraft structures has been developed. The main criterion for acceptance of a structure is a specified probability of failure. The development of this approach is motivated by the increasing use of damage-sensitive composite aircraft structures. The resulting probabilistic model takes into account a probabilistic assessment of in-service accumulated damage, the ability of non-destructive inspection methods to detect such damage, the residual strength degradation of damaged structure, quality of repair and loads/temperatures expected between damage initiation and detection/repair. The special attention has been paid to the availability of input probabilistic data and the possibility to obtain such data at reasonable cost. Computing tools and algorithms for the probabilistic analysis have been developed. The validity of the approach has been demonstrated on several existing structural components. The main result of the study is that the reliability of a damage-tolerable composite structure can be assessed on a quantitative basis, allowing aircraft manufacturers, operators and flight certification authorities to establish the maintenance service guidelines and estimate the reasonable inspection intervals. Engineers can use this methodology in the future as a guide to establish design and inspection criteria while considering structural risk and maintenance cost at the same.
\end{abstract}

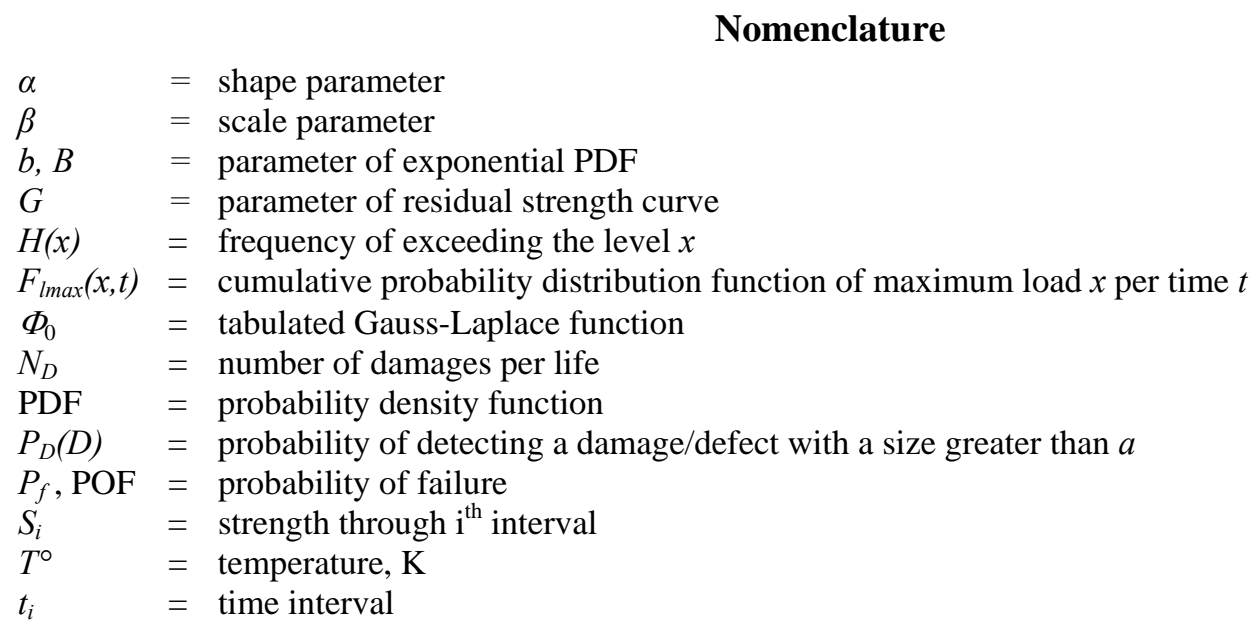

\section{Introduction}

ircraft reliability and risk engineering analysis methods and design tools have been developed for more than 30
aircraft programs, because many key engineering parameters are in fact probabilistic in nature like material property
values, gust load, damage size, etc. Although probabilistic technology offers an advanced approach to design, it
contrasts significantly with the classical safety factor deterministic design process. For this reason, design
organizations have been very reluctant to adopt even standard probabilistic methods or include them as part of their

* Professor, Department of Aeronautics and Astronautics, Box 352400, AIAA Associate Fellow.

${ }^{\dagger}$ Acting Assistant Professor, Department of Aeronautics and Astronautics, Box 352400. 
analysis capability. Other reasons cited are the complexity of failure modes, limited damage databases, and safety issues.

Current aircraft design and certification philosophies are based on deterministic approaches which use safety and knockdown factors for various design conditions such as moisture, temperature, loading, and damage. Because of higher scatter in composite material properties and sensitivity of composite structures to impact damage, traditional methods have led to very conservative designs and service guidelines for composite structures. In essence, the current approach assumes that a "worst-case scenario" for each design condition occurs simultaneously. The result is a substantial weight and cost penalty which reduce the durability, lightweight, and performance advantages of composites. $^{2}$

As the use of composite materials in aircraft structures are becoming more widespread, a greater need exists to determine the reliability of aircraft structures subject to accidental damage. Foreign object damage (FOD), ground vehicle collisions and lightning strikes are but a few examples of accidental damage that an aircraft structure must face during its operational lifetime. By using a nondeterministic approach, the structural failure risks associated with accidental damage events can be assessed quantitatively, allowing aircraft manufacturers, operators, and flight certification authorities to better evaluate and predict the damage tolerance and safety of an aircraft structure.

The primary candidate package for solving this problem has been the most advanced NASA/SwRI product IPACS-NESSUS software ${ }^{7-19}$. However the study of its capabilities has been revealed that the advanced FPI methods incorporated into this software do not work with the discrete variables such as the number of damages or the number of inspections to detect damage. The other candidates have been the "Level of Safety" method proposed by Lin, et al. ${ }^{5}$ and "Probabilistic Design of Composite Structures" method by Styuart, et $\mathrm{al}^{20}$. The approach presented here combines those two methods. However the NESSUS package has been used in a current study for obtaining the probabilistic characteristics of initial and residual strength.

The reliability assessment method presented in this paper enables the utilization of service experience to take full advantage of the benefits of using composites. The potential of this method to increase structural safety and reduce maintenance cost is not however, limited to composite structures.

Past reliability and structural risk studies have focused on fatigue of aging aircraft, which is mainly an issue unique to metal structures. Composite structures are fatigue and corrosion resistant, but are much more sensitive to damage threats because of brittle behavior during failure. Furthermore, there may be no visible evidence of damage to composite structures, even though significant internal damage has been sustained. Many probabilistic methodologies incorporating micromechanics, laminate theory, manufacturing defects, operating environment, and impact damage have been proposed for composite structures, but few however, have addressed the important issues of inspection intervals and damage detection capability.

The present study is based on a probabilistic failure analysis with the consideration of parameters such as inspection intervals, statistical data on damages, loads, temperatures, and damage detection capability, residual strength of the new, damaged and repaired structures. The inspection intervals are formulated based on the probability of failure of a structure containing damages and the quality of a repair.

\section{Reliability Formulation}

Modern damage tolerance philosophies require that damage accumulated during the service life of a component be detected and repaired before the strength of the component is degraded beyond some design threshold. The following simple example describes some fundamental concepts of probabilistic structural analysis and design: the assessment of the probability of failure for deterministically defined residual strength history as shown schematically in Fig 1. Let us assume that every structural component in an infinite fleet has this residual strength $R$ history. The initial $\mathrm{R}$ is equal to 1.5 . At the instant $t_{0}$ the damage of size $D$ occurs and $R$ is decreased to the value 1.1. At the time instant $t_{l}$ the damage is repaired and strength fully restored. There is three intervals $t_{i}$ of constant strength $S_{i}$. Then the failure may happen only because of the random external load exceeding the structural strength. The probability of failure per life is expressed as

$$
P_{f}=1-\prod_{i=1}^{N=3}\left[1-P_{f}\left(S_{i}, t_{i}\right)\right]
$$

where $P_{f}\left(S_{i}, t_{i}\right)$ is a probability of failure per $\mathrm{i}^{\text {th }}$ interval. 


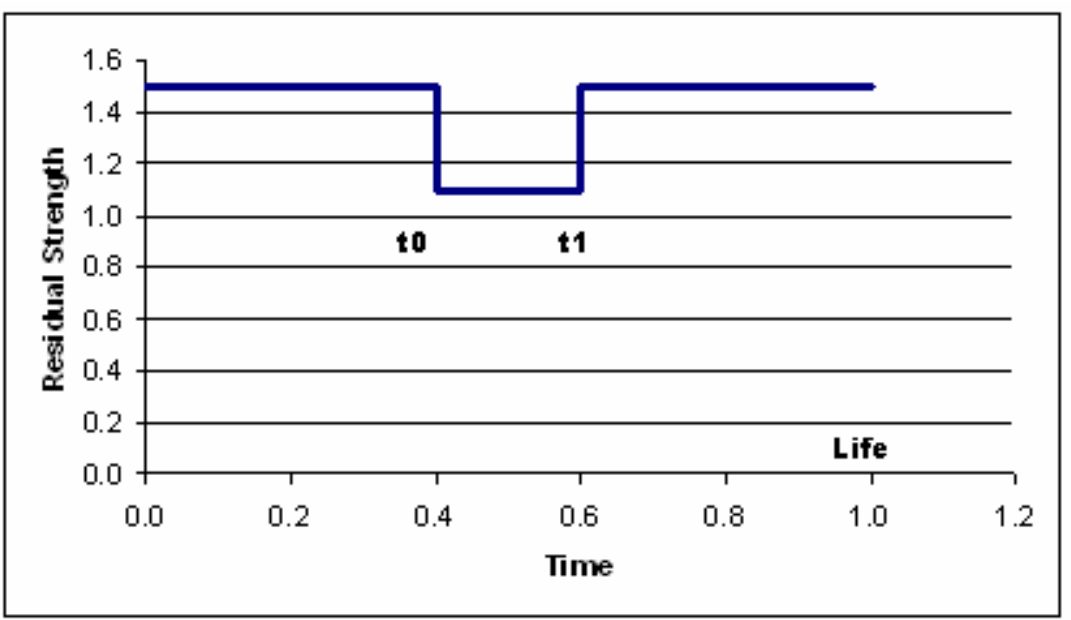

Figure 1. Random Residual Strength Life History

Let us assume the load exceedance curve looks like that in Figure 2.

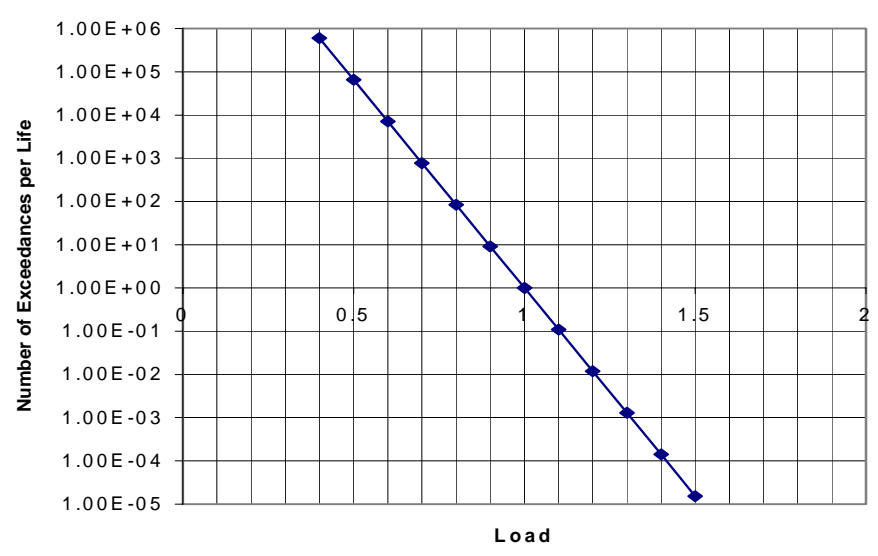

\section{Figure 2. Load Exceedance Curve}

The cumulative probability distribution function of maximum load per time $t_{i}$ may be written as [16]

$$
F_{l \max }\left(S_{i}, t_{i}\right)=e^{-H_{t}\left(S_{i}\right) t_{i}}
$$

It is easy to find from Figure 1, Figure 2 and (1.2) that $P_{f}\left(S_{1}, t_{l}\right)=P_{f}\left(S_{3}, t_{3}\right)=6.1210^{-6}, P_{f}\left(S_{2}, t_{2}\right)=4.2610^{-2}$ and the summary $P_{f}=4.2610^{-2}$.

Let's make now the picture more realistic. This means:

- The number of damages per life may be more or less than one.

- There may be several different types of damage (e.g. through crack, indentation, delamination, disbonding, etc.)

- Damage occur at random time

- Damages have different sizes

- There may be several different types of inspection (pre-flight visual inspection, maintenance inspection, etc.)

- Time of damage existence (damage life) depends on the frequency of inspections and the capability of inspection to detect damage.

Fig. 3 shows one history of the random damage size vs. time. Two types of damage are supposed: Delamination and hole. The damage size realization may be converted into residual strength realization and probability of failure calculated with equation (1). Such a procedure should take into account a number of deterministic and random variables discussed in the next two sections. 


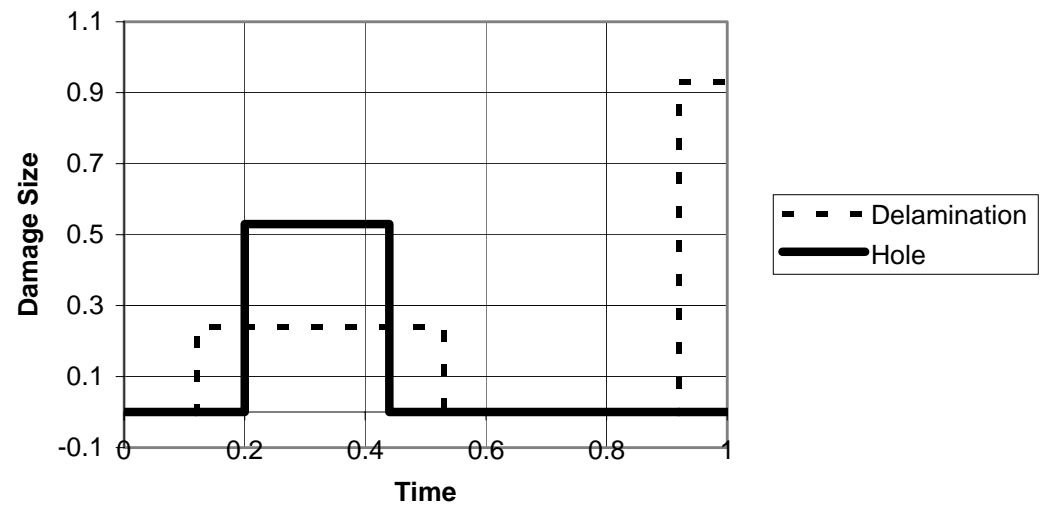

Figure 3. Damage Size Lifetime History

\section{Deterministic Variables}

In order to make the input data obtainable and simple we follow the current practice and introduce some deterministic categorization for loads/failure modes, damage types and inspection types.

\section{A. Deterministic Load cases/Failure modes}

In accordance with the majority of current structural design practices, the analysis of strength/rigidity of an aircraft structure is carried out for a finite set of conditions (e.g. various points on the V-G velocity-acceleration diagram for wing). They are called design load cases (DLC). There are many load cases, but for each particular substructure the engineer selects only a few critical cases. These cases are closely related to the potential failure modes of the structure under anticipated loading conditions. Usually this set of design cases is selected by comparing the external loads (bending moment, torque, and shear force) for various design conditions. Further, the selected set of cases is verified with finite element model. Frequently, the load cases are closely related to the segments of the flight profile. E.g. flap load cases divided into take-off and landing ones.

\section{B. Deterministic classification of defects/damages}

The information on defects/damages should be treated similarly to the load information by introduction of finite set of damage types. Different types of defect/damage may be taken like hole, delamination, surface dents, etc. The user may use his own classification. This classification should be related to the availability of methods of prediction of residual strength depending on the size of damage and the availability is the statistical data for every damage type. The selection of damage types depends also on the type of load realized in the considered site, e.g., if only tension stress takes place there, primary attention should be paid to through damage, and delamination is of minor importance.

\section{Deterministic description of inspection/repair}

There may be several types of inspection. They are distinguished by the inspection method and the inspection schedule. Currently the time of inspection is deterministic variable defined by the array if inspection times through the life. One type of inspection is always defined: pre/post-flight inspection. In case the latter is not specified in maintenance documentation, we believe that it still exists but has low resolution.

\section{Random Variables}

In considering the structural integrity of a structural component containing damages/defects, the potential random variables include number of damages, damage/defect size, time to detect/repair damage, load, and strength properties. The effectiveness of the inspections is also among the potential random variables. The effectiveness of an inspection can be characterized by its probability of detection (POD) distribution. Nine random variables are considered in the current methodology:

(1) Number of damages per life $N_{D}$, for each type of damage

(2) Time of damage initiation.

(3) Damage/defect size $D$, for each type of damage

(4) Time $t_{i}$ from damage initiation to repair that is a random function of damage size and damage schedule 
(5) Initial failure load (initial strength), for each load case

(6) Residual strength of damaged structure, for each type of damage and each load case

(7) Failure load of repaired structure, for each type of damage

(8) Structural load for each load case

(9) Structural temperatures $T^{\circ}$ at the instants when maximum external loads occurred

Most of the above mentioned variables are described by optional PDF or exceedance curve except two variables having fixed PDFs: the damage event is assumed to be a very rare and therefore the variable $N_{D}$ is described by Poisson distribution and the number of inspection to detect damage is described by geometric PDF.

\section{A. Probabilistic description of loads}

As we have already mentioned in section II, we describe the external loads in terms of exceedance curves and then use equation (1.2) to obtain the CPF of maximum load per certain time. In a modern practice of fatigue analysis, a prediction of fatigue life of most fatigue critical sites of structure is carried out by comparing the number of cycles (time) to crack initiation as obtained in laboratory fatigue tests of specimens, with cumulative damage in operation. This cumulative damage is predicted on the statistical basis as cumulative load occurrence, versus exceedance curve (Fig.2). It is not easy to obtain adequate statistical information (flight measurements) on the loads (stresses) for all structural sites of interest. In this case, the cumulative damage is predicted by using probabilistic description of governing flight parameters (e.g., maneuver load factors) or probabilistic description of atmosphere (gust) or sinking speeds at landing, etc. These parameters are well studied so that their frequency of occurrence can be predicted, if the typical missions are known. However, the local stressed state is determined by the combination of flight parameters (load factor with speed, Mach number, etc). This problem is usually solved in a following way:

All mission profiles of aircraft are divided into flight segments so that the load distribution during the one flight segment is approximately constant and load variation can be described by one governing parameter. Usually static design case distributions are attributed (in conservative manner) to these segments, e.g., pull-up maneuver, load distribution is used together with maneuver load factor (governing parameter) and frequency of occurrence. Rough air loads are described by gust velocity as the governing parameter, exceedance curve, and corresponding load distribution (cruise, flaps-down configuration, etc). Landing loads are described by sinking speed occurrence together with appropriate two-point landing load distribution.

The linear relationships between the governing load parameter and the stress in a considered location is determined from stress analysis for the corresponding design case. Applying some relevant strength criterion, the occurrence of site loads can be obtained.

In addition to exceedance curves, the proposed method and software optionally utilize some popular PDFs such as Gumbel extreme value type I, lognormal, etc.

\section{B. Probabilistic description of structural temperature}

Temperatures and load are certainly correlated variables and in general should be described by bidimensional PDF. The statistical data on structural temperature are available only for supersonic aircraft, reentry vehicles and similar. We are going to use simplified model where this correlation is hidden in different flight segments (design load case). Temperatures are closely related to the external loads and the philosophy of their description may be similar. The cumulative probability function should be obtained for each flight segment (design loading case). Within one design loading case we will consider mechanical load and temperature to be independent random variables. Then the only necessary thing to know is the cumulative probability distribution of various temperatures duration. This data may be derived on the basis of predicted design usage of aircraft.

As a rough approximation the model [19] can be also used to obtain initial CPF. According to [19], this function consists of two branches: the first is common for all planes, which characterizes the frequency of low temperatures, and the second depends on the maximum Mach number $M_{\max }$ of a given plane. This function can be approximated by the formula:

$$
\begin{aligned}
F\left(T^{\circ}\right)=0.5+\Phi_{0}\left[\frac{T^{\circ}-T_{0}^{\circ}}{\sigma_{T}}\right] \\
\text { where: } \quad \boldsymbol{T}_{0}^{\circ}=310 \mathrm{~K} ; \boldsymbol{\sigma}_{\boldsymbol{T}}=25 \mathrm{~K} \text { at } \boldsymbol{T}^{\circ}<\boldsymbol{T}_{\boldsymbol{p}}^{\circ} \quad \boldsymbol{T}_{0}^{\circ}=0 \mathrm{~K} ; \boldsymbol{\sigma}_{\boldsymbol{T}}=61+11 \boldsymbol{M}_{\boldsymbol{m a x}} \text { at } \boldsymbol{T}^{\boldsymbol{\rho}} \boldsymbol{T}_{\boldsymbol{p}}^{\circ} .
\end{aligned}
$$

Here: 


$$
T_{p}^{\circ}=\frac{310\left(61+11 M_{\max }^{2}\right)}{36+11 M_{\max }^{2}}
$$

$\Phi_{0}$ is a tabulated Gauss-Laplace function, $\quad \boldsymbol{T}$ is a boundary-layer temperature in Kelvin.

\section{Probabilistic description of defects/damages}

Available statistical data, as well as relevant mathematical models, derived on the basis of these data can be used for probabilistic description of defects/damages. Further, the damages are described either by the frequency of occurrence (exceedance) of damages of various sizes of $H_{D}(D)$, derived for each type of damage or by damage size PDF plus the Poisson distribution parameter $\lambda$ equal to the average number of damages per life. This description is very similar to that for loads.

We use two classes of mechanical damages: manufacturing defects and operational damages. The damage types within these classes are same. Defects and damages are described by similar exceedance curves, but these curves are used in different ways. Manufacturing defect is generated randomly together with initial strength in time instant $t=$ 0 . Operational damages are randomly scattered over the life using uniform PDF.

The relationship between the exceedance curve $H_{D}(D)$ for a damage/defect size and corresponding cumulative distribution function is defined as follows:

$$
F_{D}(D)=1-\frac{H_{D}(D)}{H_{D}(0)}
$$

\section{Probabilistic description of inspections}

The efficiency of inspection should be described by the probability to detect the damage of a given type and given size. This probability is shown on Figure 4. Only a few attempts to identify this function are known from the literature. As a rule, special tests are needed to obtain this probability. Having representative experts inspect, by the appropriate methods, different zones of a structure having damages of different size and type can do this. The probability of detection is determined as the ratio of a number of successful inspections to their total number. It is also possible to determine the detection probability by comparing the empirical probability function of detected damages with theoretical one, assuming that the difference between mentioned functions is the probability for detection for various sizes of damage.

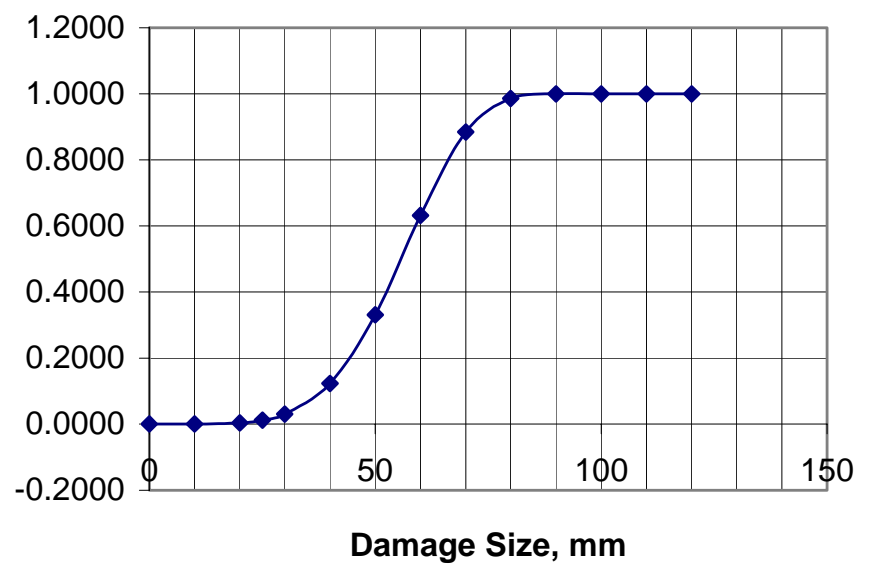

\section{Figure 4. Probability of Damage Detection per Inspection}

The random time to detect damage can be expressed as $t_{d a m}=T^{*} \xi$, where $\xi$ is a discrete random variable having the Geometric distribution. By definition, a discrete random variable $\xi$ is said to have a Geometric distribution with parameter $p>0$, if $\operatorname{Prob}[\xi=n]=p(1-p)^{n}$. 4.

In our application this parameter $p$ depends on a damage size and should be determined from the data like Figure

\section{E. Description of repairs}

There may be several decision-making algorithms, if the damage is detected. For instance: 
- If delamination size less than $2 \mathrm{~cm}$ is detected in field inspection, the repair is not necessary

- If delamination is more than $2 \mathrm{~cm}$ but less than 5 , the repair may be postponed until regular maintenance

- If delamination is more than $5 \mathrm{~cm}$, it should be repaired at once.

At present the simplest algorithm is realized in a model. If the damage is detected, there are two options: to repair it at once and to postpone to scheduled maintenance. The method of repair is directly determined by type of inspection, which has resulted in the detection of damage. The degree of strength recovery in repair is determined by the method of repair corresponding to inspection. This degree of strength recovery is described as follows:

Assume that we made a number of same design composite components, simulate the damages of equal type, size and location in them, repair those damages with the chosen method, then test the components and obtain an average failure load and failure load variance. We will describe the strength after repair by the "strength recovery coefficient" which is equal to the ratio of this average failure load to that of a new structure and the coefficient of variation of the strength after repair.

It was found that this logic does not strongly influence the final result. However it is possible to devise the logic which considerably influences the POF.

\section{Computational Methods}

As it has been mentioned earlier, the problem of reliability of damage-tolerant composite structures is solved here by computer simulation. Several sampling-based probabilistic methods have been considered as candidates to solve this problem such as standard Monte-Carlo method (MC), and various methods based on Fast Probability Integration concept. The latter includes first and second order reliability methods (FORM, SORM), various importance sampling methods and advanced mean-based methods. It was found that most popular FPI methods work well with smooth performance functions (g-functions), but are not appropriate in case of its discontinuity. As the model considered here uses some discrete variables and variables with truncated PDF, main attention has been paid to MC simulation. It is well-known that MC provides accurate results but is time-consuming especially if the gfunction is evaluated through finite-element model with many degrees of freedom. Fortunately, the MC simulation realized for this model shows rather short computation time less than 10 seconds for a reasonable POF level from $10^{-3}$ to $10^{-5}$ per life on modern PC. This is generally enough for parametric analyses.

Two close methods have been developed depending on the realization of g-function: S-L (Strength-Load) method and POF method. The methods have a common part consisting of simulation of residual strength histories (RSH). Each simulated RSH consists of a sequence of intervals with constant strength like ones shown in Figure 1. In S-L method the random load and random temperature are generated for each interval and compared with the residual strength. Then the failure limit state is evaluated for each random sample. If for $\mathrm{L}>\mathrm{S}$, the failure is recorded. The ratio of the number of structural lives with failure to the total number of simulated lives is the POF. In POF method, the probability of failure is calculated in a way explained in section II. The average value and standard deviation of POFs are calculated and the number of sampled histories is selected to satisfy the accuracy requirements. This latter method reduces the size of the analysis region and generally is signicantly more efficient than S-L simulation. The advantage of S-L method is that the description of every failure may be recorded. If the model is complicated (many load cases, damage types, inspection types) this feature may help to understand the situation more than sensitivity analysis.

\section{A. RSH simulation}

Each residual strength history is based on a number of constant damage size intervals. The starting time of each interval is random value and the length is a random function of the probability of damage detection and inspection interval. The history may be randomly simulated using finite set of random variables such as damage occurrence rate and probability of damage detection. In other words we have to create a generator of random functions characterized by multivariate PDF $f(\boldsymbol{v})$, where the components of a random vector $\boldsymbol{v}$ are the variables mentioned in section IV. 


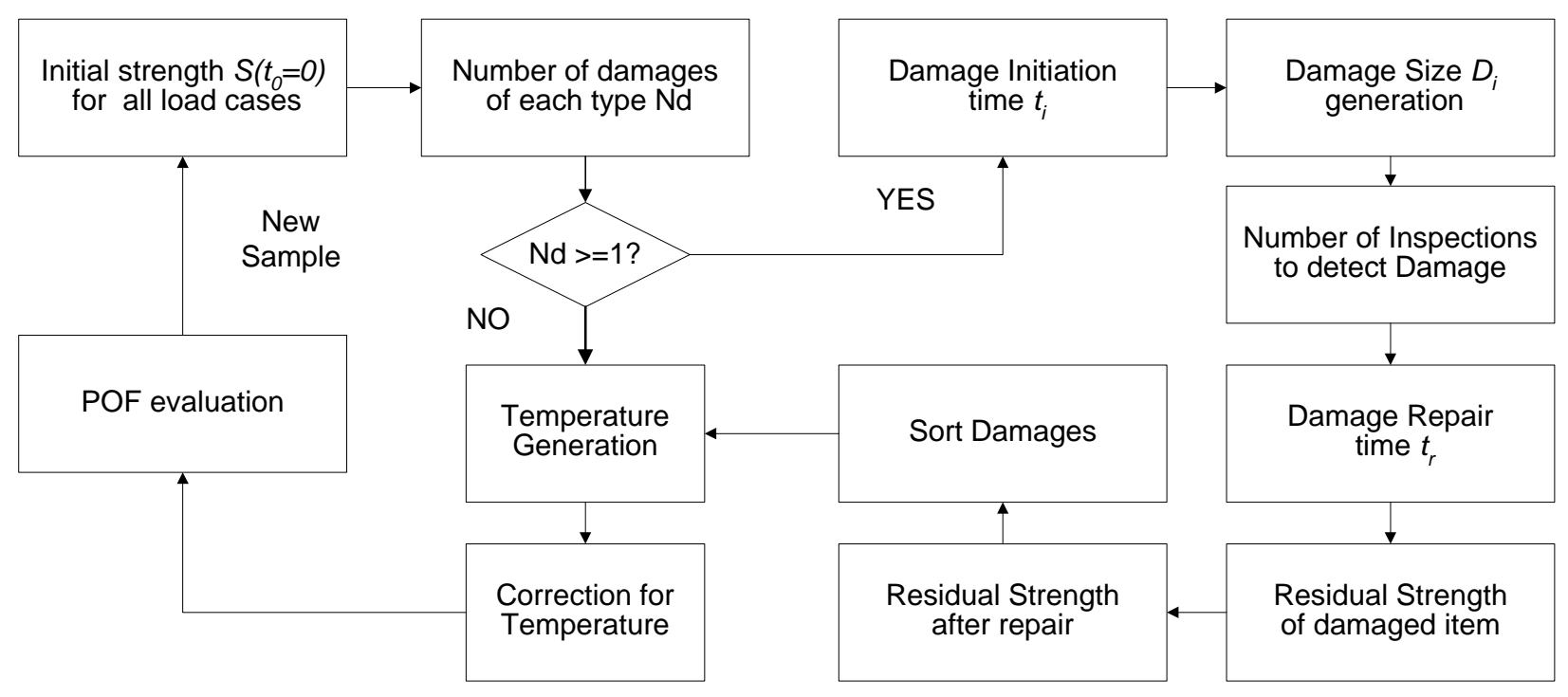

Figure 5. Simulation flowchart

The generation scheme is illustrated in Figure 5. The sequence of one residual strength history generation is as follows:

1) Each random vector generation begins with the generation of initial strength values $\left(t_{0}=0\right)$ for all considered load cases.

2) The numbers of defects and damages of all types (if any) are generated

3) If no manufacturing defects and operational damages occur during this life, random temperatures of structure at the instants of maximum load occurrence are generated for all DLC.

4) The initial strength values, $S\left(t_{0}=0\right)$, are corrected depending on temperature and POF is evaluated for time equal to life and the new generation cycle starts.

5) If the total number of defects and damages is more than zero, sizes of manufacturing defects of all types are generated, followed by generation of residual strength values, $S\left(t_{i}\right)$ for all DLC. The manufacturing defects are listed in a damage list and after that treated as operational damages occurring at $t_{0}=0$.

6) Operational damages are scattered over the life using a uniform distribution generator. The time instants, $t_{i n}$, are generated and added to the list of damages.

7) The values of damage size are generated.

8) For each damage, the time of detection and time of repair are randomly generated using the probability of detection and inspection schedule. The random numbers of inspections of different types of damage detection are generated from geometric PDF, and then these inspections are found in the inspection schedule. The time of detection is the shortest time between randomly simulated times for different types of inspection. In this way it determines repair time instants and the time of damage existence.

9) The values of residual strength with damage $S\left(t_{i}\right)$ are generated.

10) As the result of previous actions we have a set of intervals with constant strength. It may have intact structure interval, overlapping intervals of damaged state, and repaired state intervals. Here the special procedure is applied to sort intervals and eliminate possible overlapping. This procedure results in new sequence of intervals, characterized by length $t_{i}$ and level of residual strength $S_{i}$. The sequence includes also intact structure interval and repaired structure intervals (if any).

11) The residual strength values, $S_{i}$, are corrected depending on temperature.

12) $P_{f}$ is evaluated using relations (1.1-1.2) and the new generation cycle starts.

\section{Demonstration cases}

\section{A. Example problem 1}

The input data for this problem are typical for current damage-tolerant composite aircraft parts. This structure complies with the requirements of AC 20-107A. The main assumptions are listed below:

1) We assume just one loading case. The load exceedance curve per life is expressed as $H_{t}(x)=H_{0} \exp (-x / b)$, where $H_{0}=4.2683 \mathrm{e} 9 ; b=0.112742$. These parameters are obtained on the basis of observations published 
by J.Taylor ${ }^{22}$. The frequency of $1 \mathrm{~g}$ level crossings is 60 times per flight hour. The frequency of limit $2.5 \mathrm{~g}$ level crossings is one per life (10000 flights).

2) Here we assume only one type of damage. It is known that the size of dust and sand particles, hailstones, and raindrops in atmosphere follows in general the exponential distribution. So it is quite natural to assume exponential distribution of initial impact damage size. The exceedance curve will have the simple shape

$$
H_{D}(D)=E_{0} e^{-D / B}
$$

If we take two points $P_{I}\left(D_{1}\right)$ and $P_{2}\left(D_{2}\right)$ of detected damage exceedance curve, which are in the region of $100 \%$ detected damage and assume that exceedances of initial and detected damages are equal, we can get the following expressions:

$$
e^{-\left(D_{1}-D_{2}\right) / B}=\frac{P_{1}}{P_{2}} ; B=\frac{D_{2}-D_{1}}{\ln \left(P_{1} / P_{2}\right)}
$$

Substituting the data obtained by $\operatorname{Lin}^{5}$, we can obtain $\mathrm{B}=1.6-1.8 \mathrm{in}$. In this example $B=1.5$ in and $E_{0}=1$ (one damage per life on average) are used.

3) We assume here just one type of inspection. Probability of damage detection is described by Weibull function ${ }^{5}$.

$$
\begin{aligned}
& P_{D}(D)=1-\exp \left(-\frac{D}{\beta}\right)^{\alpha} \\
& \alpha=1.4 ; \quad \beta=1.64 \text { in }
\end{aligned}
$$

4) The initial average value of strength is equal to $2.5 \times 1.5 \times 1.415=5.3$. The latter factor is supposed to be an additional margin of safety for composite structure in a considered loading case. The strength scatter is described by the Gauss PDF with the coefficient of variation $\mathrm{C}_{\mathrm{V}}=5 \%$, that is the same for both initial strength and strength of damaged structure. Relative reduction of the residual strength vs. damage size D is described by the function

$$
\begin{aligned}
& Y(D)=A+(1-A) \exp \left(-\frac{D}{G}\right) \\
& A=0.55 ; \quad G=2.0
\end{aligned}
$$

All requirements of AC 20-107A are satisfied.

5) The strength after repair is described by the Gauss PDF with the average equal to $100 \%$ of initial strength and coefficient of variation $\mathrm{Cv}=5 \%$.

6) The strength is considered to be independent on temperature.

Figure 6 shows a good agreement of the Probability of Failure evaluated with two considered methods for the above mentioned input data.

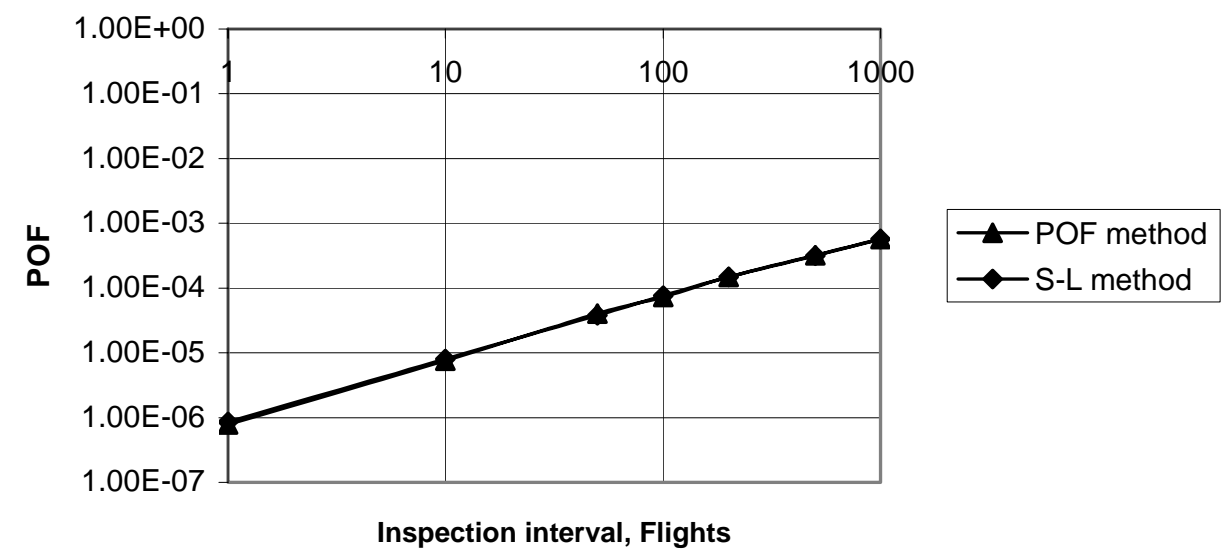

Figure 6. Comparison of POF and S-L methods 
This analysis shows, that in order to provide POF on the level of 0.0001 , we have to apply an additional factor of safety 1.415, have a strength coefficient of variation around $5 \%$ and inspect our structure every 100 flights.

\section{Parametric study}

In general, POF and corresponding Inspection Interval depend on all input parameters. The most important have been selected for this particular case. In all cases the inspection interval has been determined as one corresponding to Probability of Failure about $10^{-4}$ per life.

a) Effect of Various Extensions of Load Exceedance Curve

The PDF for maximum load is determined via exceedance curve of external loads per flight hour. The most reliable method for deriving the load exceedance curves is to measure loads in flight. The exceedance curve is usually known in the range from the load of level flight to that at the limit load factors. For the purpose of reliability assessment, the reasonable extrapolation of this curve to the region between the limit and ultimate load is needed. Figure 7 shows three possible extensions of the Load Exceedance Curve and Figure 8 shows the effect of those extensions. Extension 1 is suitable if the load source is the atmospheric turbulence; extension 3 is more suitable for maneuver load when it is essentially restricted, e.g., aileron deflection; extension 2 may be good for combination of 1 and 3.

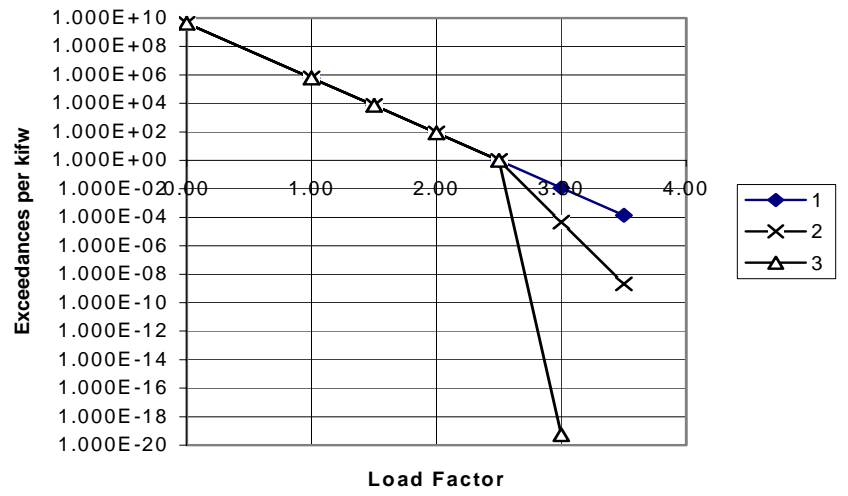

Figure 7. Various Extensions of Load Exceedance Curve 


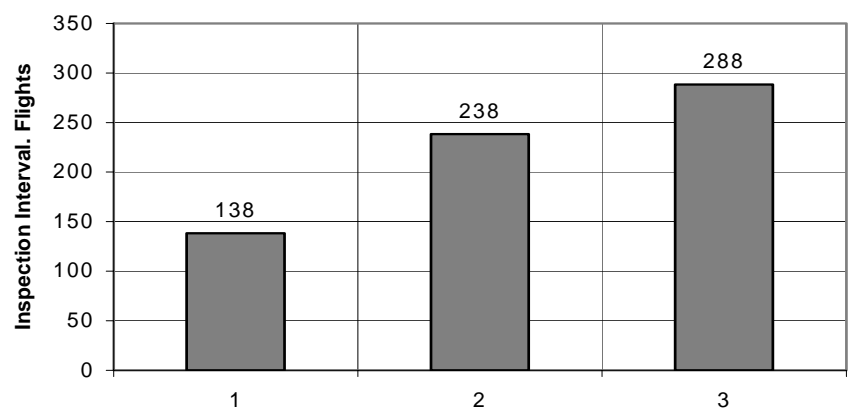

Figure 8. Effect of Various Extensions of Load Exceedance Curve

b) Effect of Damage exceedance curve variations

The Damage exceedance curve in this example problem is described by the exponetial function (4). The Effect of Damage Exceedance Intercept $E_{0}$ is shown on Figure 9. The effect of Damage Exceedance Slope is shown on Figure 9.

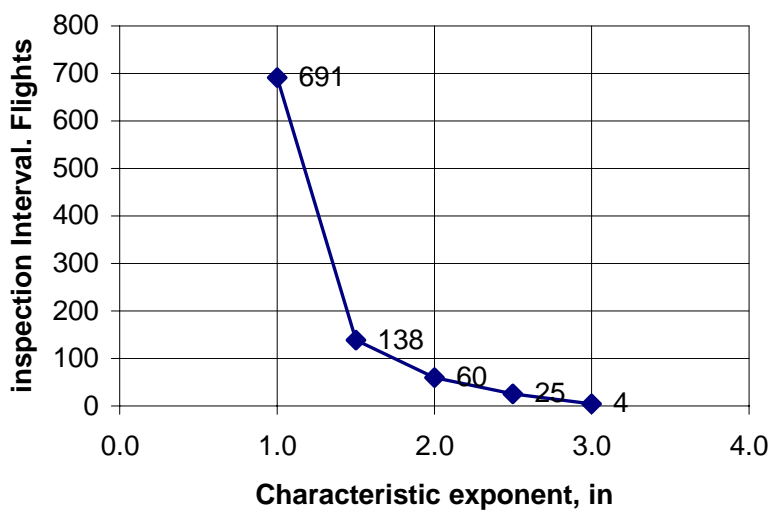

Figure 9. Effect of Damage Exceedance Slope

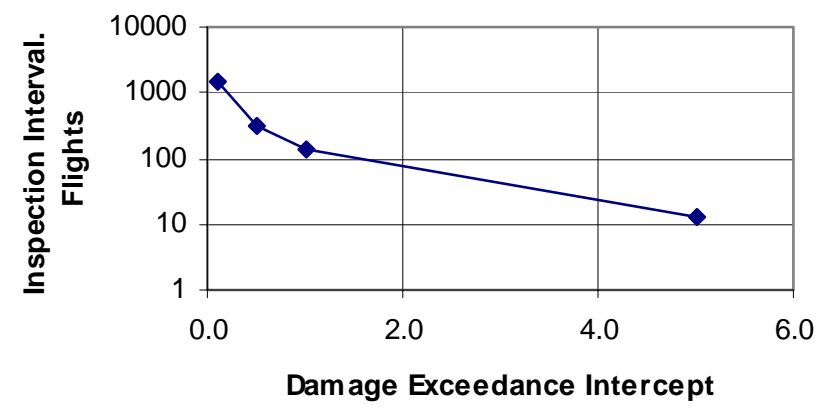

\section{Figure 10. Effect of Damage Exceedance Intercept}

c) Effect of Average Detected Damage

In this example, the probability of damage detection is described by Weibull function. The influence of Weibull scale parameter is shown on Figure 11. 


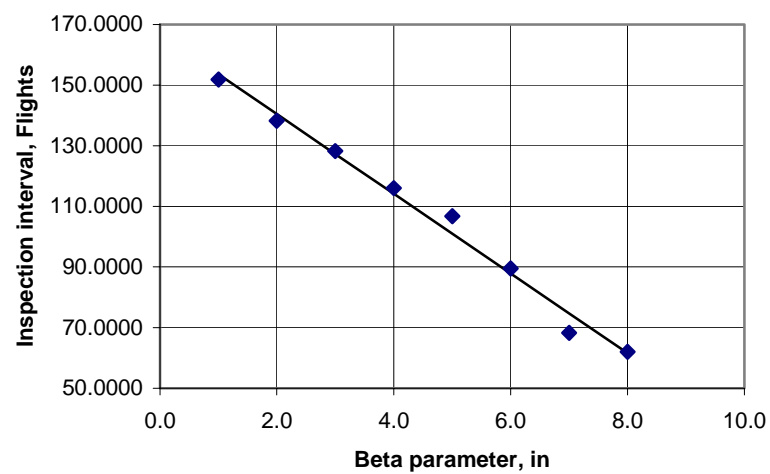

\section{Figure 11. Effect of Average Detected Damage}

d) Effect of Residual Strength vs. Damage Size variations

In order to describe Residual Strength vs. Damage Size, we use here the relation(5). The Effect of Residual Strength asymptote A is shown on Figure 12.

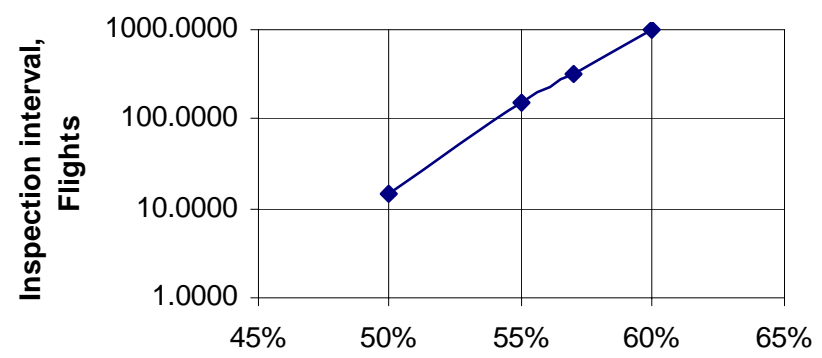

Residual Strength Asymptote, \% of Initial

Figure 12. Effect of Residual Strength asymptote

The Effect of Residual Strength slope G is shown on Figure 13.

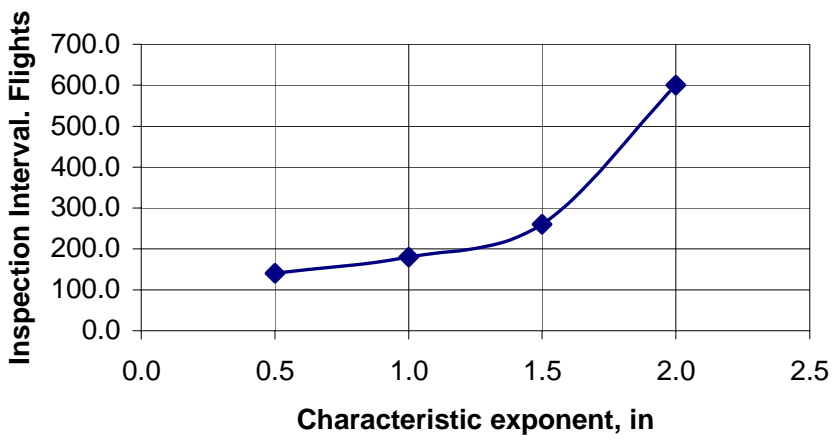

\section{Figure 13. Effect of Residual Strength slope}

\section{e) Effect of Strength Recovery after Repair}

The importance of strength restoration after repair is shown Figure 14. Strength Recovery characterizes the quality of repair. It depends only on the method of repair. But the probability of failure depends (in general) on Strength Recovery \%. The higher this \% is, the lower the POF should be. We determine the Inspection Interval at const level of POF $=10^{-4}$ per life $\left(10^{-8}\right.$ per flight). If we have perfect repair method (Strength Recovery $\left.\%=100\right)$, we obtain the POF $=10^{-4}$ per life, when the Inspection Interval is equal to 130 flights. If our repair method restores only $80 \%$ of the initial strength, we have to inspect the structure more frequently to obtain the same $P O F=10^{-4}$ per life. But if our repair restores only $65 \%$, we can not reach POF $=10^{-4}$ per life, even if we inspect after each flight. 
Assume that we repair some damage, then test the structure and obtain the failure load equal to only $90 \%$ of a new structure. Probably we may say that this is a bad repair method and it will increase the probability of failure. But calculations of POF show that this is not true, that this structure is quite insensitive to the repair quality, because all failures happen when our structure is damaged (not repaired yet). The quality of repair influences the POF and Inspection Interval only if the Strength Recovery \% is less than 80.

The conclusion is that we can use this "bad" repair method. This is not trivial and quite important conclusion. This means that we do not care much about repair quality. We do not have to pay much money to invent "good" repair method and/or apply some expensive repair method. Obviously, this is not a general rule. This depends mostly on the behavior of the residual strength vs. damage size.

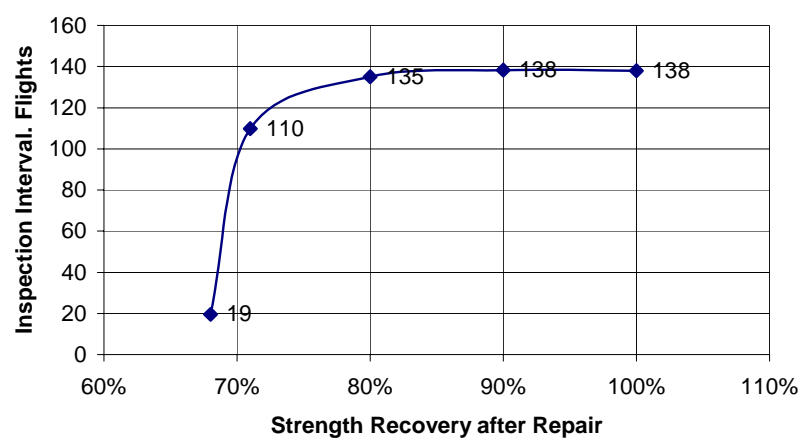

Figure 14. Effect of Strength Recovery

\section{B. Example problem 2: Composite fin}

The input data for this problem are taken from FAA report ${ }^{19}$. This example looks quite good for comparing the R-S method with POF method.

The main features of the problem are:

1) The damage rate is quite low (0.231 per life)

2) The load cases include one subsonic and one supersonic case with elevated temperatures

3) Two types of damage are considered: delamination and hole or crack

4) Two types of inspection are considered: pre/post flight (type 1) and special (type 2) inspection method applied during maintenance. The detection probabilities are shown in Figure 15.

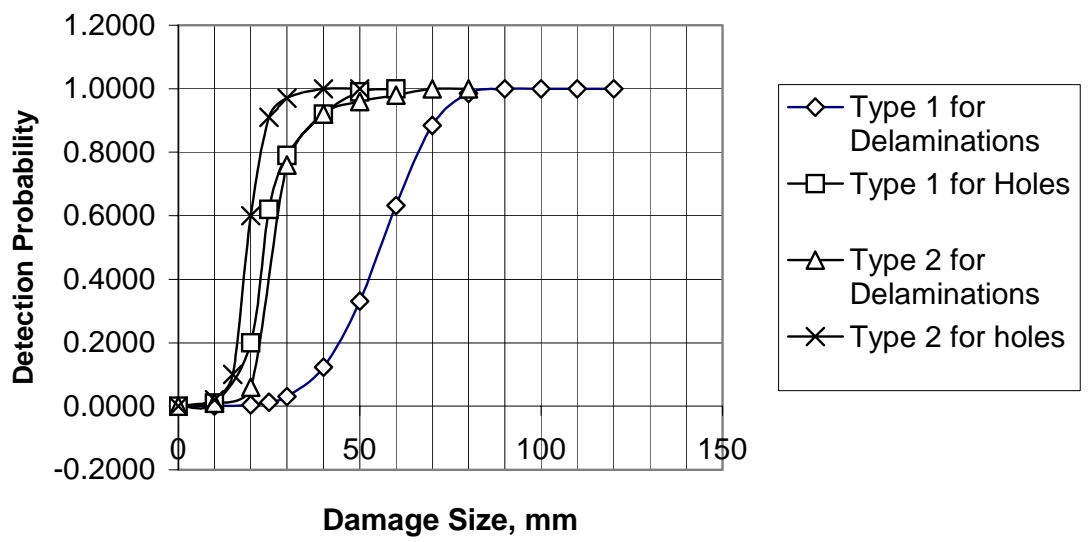

\section{Figure 15. Detection Probability vs. Damage Size}

The analysis shows that most trends are similar to ones for previous problem. It is worth to mention that POF almost does not depend on the inspection interval of the second type inspection because the majority of damages are detected with the type 1 inspection. Some specific results of parametric study are shown in Figures 16 through 18. The POF obtained for this composite fin is rather high. We can expect that about $1 \%$ of fin composite panels may fail within their usage time. Nevertheless the designer agreed to pay this tribute to mechanical damages because it was supposed that this failure if happens would not lead to catastrophic structural failure. The fin has two metal spars, which carry an essential part of external load. This aircraft has two main fins and two additional dorsal fins which guarantied the successful flight completion is case of one composite panel failure. 


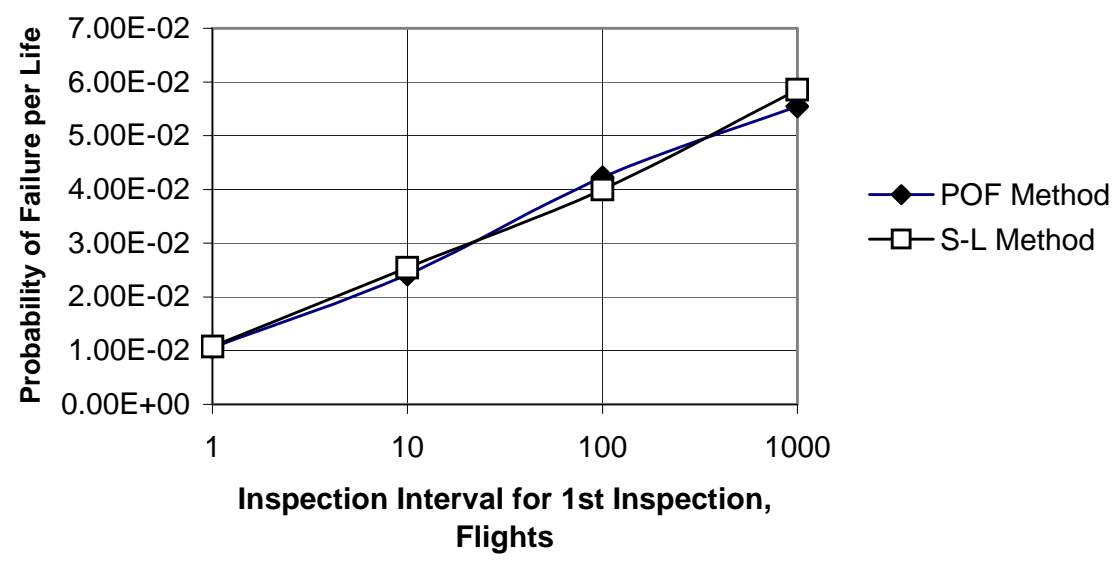

Figure 16. POF vs. Interval Comparison

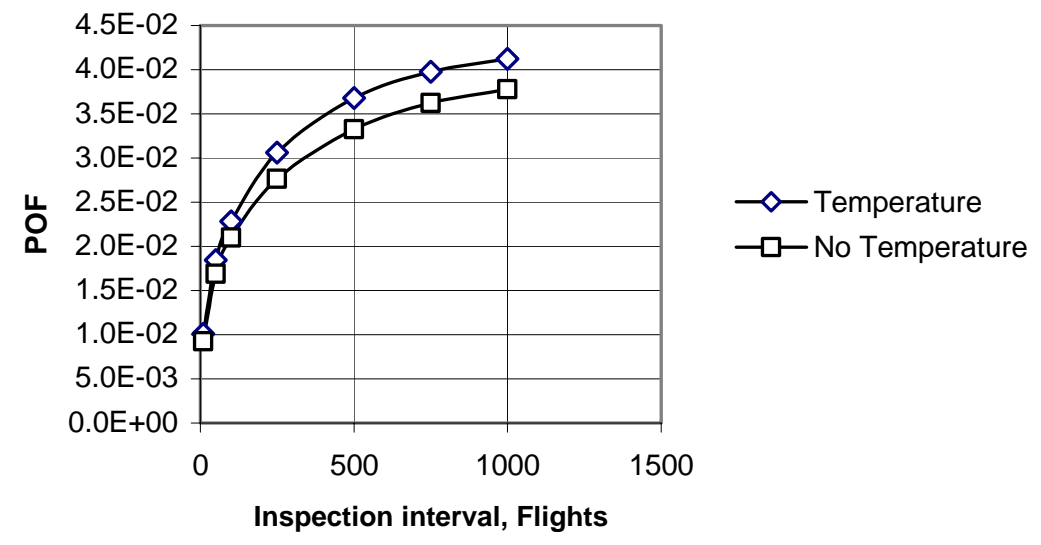

Figure 17. Effect of Temperature on POF

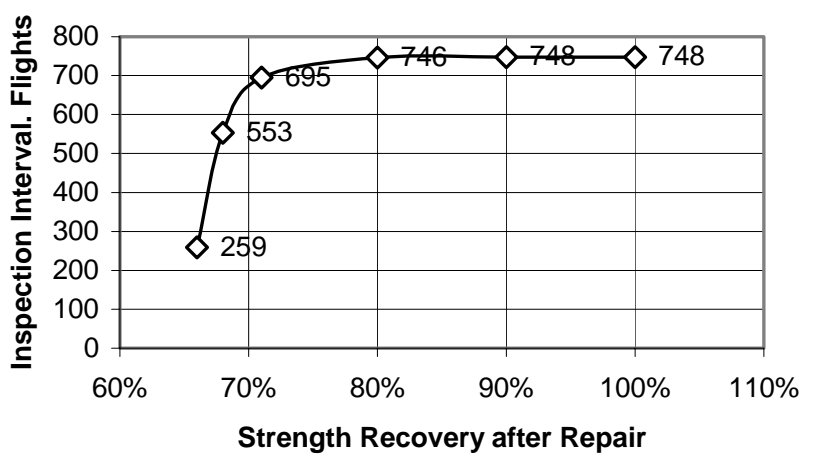

Figure 18. Effect of Strength Recovery after repair. 


\section{Conclusions}

A method to quantify the damage tolerance design and reliability for aircraft composite structures in a presence of multiple uncertainties was presented. The main focus of the method is to establish the rational inspection interval that allows maintaining high structural reliability. The efficiency of the method is illustrated for two typical aircraft composite structure models. The following observations can be mentioned:

1) Probabilistic methods may provide the basis for quantifiable analysis of reliability and inspection intervals for damage-tolerant composite aircraft structures and allow aircraft manufacturers, operators and flight certification authorities to establish the maintenance service guidelines to reduce lifecycle cost.

2) The inspection interval that insures the reasonably high reliability depends primarily on statistical characteristics of external loads, damage rate and residual strength of damaged structure.

3) The behavior of the load exceedance curve near and higher than the limit or the scatter of maximum load per life, has a large influence on the reliability and inspection intervals and should be a subject of special attention when applying the probabilistic methods.

4) Due to the lack of statistical data, the most uncertain variables in a probabilistic damage tolerance design are the damage size and frequency. Unfortunately, available in-service data may not contain complete descriptions of damage size, structural locations and inspection method.

5) The extent of strength recovery after repair does not influence the reliability and inspection interval in a broad range of values.

\section{Acknowledgments}

This work was supported under Federal Aviation Administration Research Grant "Development of ReliabilityBased Damage Tolerant Structural Design Methodology ". Peter Shyprykevich is the Grant Monitor. The authors wish to thank the FAA Center of Excellence at the University of Washington (AMTAS) for sponsoring the current research project. Thanks also to Dr. Cliff Chen of Boeing, for the invaluable discussions in the development of the reliability method presented. 


\section{References}

1 Tong, Y. C., “Literature Review on Structural Risk and Reliability Analysis," Aeronautical and Maritime Research Laboratory, Rept. DSTO-TR-1110, Melbourne, Australia, Feb. 2001.

2 Rusk, D. T., "Reliability-Based Methods Applied to the Design of Damage Tolerant Aircraft Structures,” M.S. Thesis, Dept. of Aeronautics and Astronautics, Univ. of Washington, Seattle, WA, June 2000.

3 Long, M. W., and Narciso, J. D., "Probabilistic Design Methodology for Composite Aircraft Structures,” U.S. Dept. of Transportation/Federal Aviation Administration, Rept. DOT/FAA/AR-99/2, Washington, DC, June 1999.

4 Gray, P. M., and Riskalla, M. G., “Development of Probabilistic Design Methodology for Composite Structures,” U.S. Dept. of Transportation/Federal Aviation Administration, Rept. DOT/FAA/AR-95/17, Washington, DC, Aug. 1997.

5 Lin, K. Y., Du, J. and Rusk, D. T., “Structural Design Methodology Based on Concepts of Uncertainty,” National Aeronautics and Space Administration, Rept. CR-2000-209847, Langley, VA, Feb. 2000.

6 Rusk, D. T., Lin, K. Y., Swartz, D. D., and Ridgeway, G. K., "Bayesian Updating of Damage Size Probabilities for Aircraft Structural Life-cycle Management,” AIAA Journal of Aircraft, Vol. 39, No. 4, July 2002, pp. 689-696.

7 Roach, D., and Rackow, K., "Improving In-Service Inspection of Composite Structures - It's a Game of CATT and MAUS,” U.S. Dept. of Defense/National Aeronautics and Space Administration/Federal Aviation Administration Aging Aircraft Conference, Sept. 2003.

8 Chamis, C. C. and Shiao, M. C., .Structural Reliability, Risk and Certification: Computational Simulation,. in Computational Structural Mechanics and Multidisciplinary Optimization, R. V. Grandhi, W. J. Stroud, and V. B. Venkayya (editors), ASME, AD-Vol. 16, 1989, pp. 27-34.

9 Minnetyan, L., Chamis, C. C., and Murthy, P. L. N., Damage and Fracture in Composite Thin Shells, NASA TM-105289, October 1991.

10 Chamis, Christos C., .Computer Codes Developed and Under Development at Lewis, In Computational Structures Technology for Airframes and Propulsion Systems, A. K. Noor, J. M. Housner, J. H. Starnes, Jr., D. A. Hopkins, and C. C. Chamis (compilers), NASA CP-3142, 1992, pp. 43-57.

11 Chamis, Christos C., .An Overview of Computational Simulation Methods for Composite Structures Failure and Life Analysis,. in Computational Methods for Failure Analysis and Life Prediction, A. K. Noor, C. E. Harris, J. M. Housner, and D. A. Hopkins (compilers), NASA CP-3230, 1993, pp. 205-223.

12 Murthy, P. L. N., Chamis, C. C., and Singhal, S. N., "Hierarchical Nonlinear Behavior of Hot Composite Structures", In Mechanics of Composite Materials: Nonlinear Effects, M. W. Hyer (editor), ASME AMD-Vol. 159, 1993, pp.233-244.

13 Chamis, Christos C., Damage Tolerance and Reliability of Turbine Engine Components, NASA TP-1999-209878, 1999.

14 Riha, D.S. Thacker B.H., Hall D.A.,. Auel T.R, and S.D. Pritchard, "Capabilities and Applications of Probabilistic Methods in Finite Element Analysis," Int. J. of Materials \& Product Technology, Vol. 16, Nos. 4/5, 2001. Also Proc. Int. Soc. Science and Applied Technologies (ISSAT) Conf. on Reliability and Quality in Design, Las Vegas, Nevada, 11-13 August 1999

15 Riha, D.S., Thacker, B.H., Millwater H.R., Wu, Y.-T. and Enright, M.P., "Probabilistic Engineering Analysis Using the NESSUS Software," by 41st Structural Dynamics and Materials Conference, Paper 2000-1512, Atlanta, Georgia, April 2000.

16 Thacker, B H., Nicolella, D. P., Kumaresan, S., Yoganandan, N., Pintar, F. A., "Probabilistic Finite Element Analysis of the Cervical Spine”, Math. Modeling and Sci. Computing, Vol. 13, No. 1-2, pp. 12-21, 2001.

17 Thacker, B.H., Riha, D.S., Millwater, H.R., and Enright, M.P., "Errors and Uncertainties in Probabilistic Engineering Analysis," Proc. AIAA/ASME/ASCE/AHS/ASC 42nd Structures, Structural Dynamics, and Materials (SDM) Conf., AIAA 2001-1239, Seattle, WA, 16-19 April 2001.

18 Thacker, B.H., Rodriguez, E.A., Pepin, J.E., and Riha, D.S., “Application of Probabilistic Methods to Weapon Reliability Assessment”, Proc. AIAA/ASME/ASCE/AHS/ASC 42nd Structures, Structural Dynamics, and Materials (SDM) Conf., AIAA 2001-1458, Seattle, WA, 16-19 April 2001

19 Riha, D.S., Thacker, B.H., Enright M.P., Huyse, L. and Fitch, S.H.K., "Recent Advances of the NESSUS Probabilistic Analysis Software for Engineering Applications," Proc. AIAA/ASME/ASCE/AHS/ASC 42nd Structures, Structural Dynamics, and Materials (SDM) Conf., AIAA-2002-1268, Denver, CO, 22-25 April 2002

20 Ushakov A., Stewart A., Mishulin I., and Pankov A. "Probabilistic Design of Damage Tolerant Composite Aircraft Structures”, U.S. Dept. of Transportation, Federal Aviation Administration, Report DOT/FAA/AR-01/55, Washington DC, January 2002.

21 Stuchalkin Yu., Stewart A., and Ushakov A., "Characteristics of Certification of Composite Structures”, Chapter 7, Topics in Composite Materials in Aerospace Design, Ed. Zagainov G., Chapman \& Hall Publishers in Science, Technology, Medicine, and Business, London, 1995

22 Wu Y.-T., Enright M. P., and Millwater H. R., "Probabilistic Methods for Design Assessment of Reliability with Inspection” AIAA JOURNAL Vol. 40, No. 5, May 2002 pp. 937-946.

23 Taylor, J., "Manual on aircraft loads", Oxford, New York, Published for and on behalf of Advisory Group for Aeronautical Research and Development, North Atlantic Treaty Organization by Pergamon Press, 1965 